\title{
ALL-OPTICAL APPROACH FOR CONVERSION OF A BINARY NUMBER HAVING A FRACTIONAL PART TO ITS DECIMAL EQUIVALENT AND VICE-VERSA
}

\author{
SOMA DUTTA* and SOURANGSHU MUKHOPADHYAY ${ }^{\dagger}$ \\ Department of Physics, The University of Burdwan \\ Burdwan, pin-713104, West Bengal, India \\ *soma.dtta@gmail.com \\ †sourangshu2004@yahoo.com
}

Received 25 October 2010

\begin{abstract}
Optics is found as a very potential candidate in information processing and computing. Several all optical methods have been proposed for implementation of all optical logic and arithmetic devices during the last few decades. In this regard, the role of optical tree architecture can be mentioned as an important approach for conversion of an optical data from binary to decimal and vice-versa. In this communication, the authors propose a new concept of conversion of a binary number having some fraction (fractional value) to its equivalent decimal counterpart and its vice-versa. To perform this operation, optical tree and some nonlinear material based switches are used properly.
\end{abstract}

Keywords: Non-linear optics; optical computation; optical tree-architecture.

\section{Introduction}

Optics has already been established as a potential and promising candidate in information and data processing. Many all-optical logical, algebraic and arithmetic processors have been proposed since the middle of the decade of seventy. ${ }^{1-5}$ Several schemes of all optical logic gates, optical digital memory units, optical algebraic processors, image processors etc were proposed by scientists and technologists. ${ }^{6-11}$ The choice of optical signal in replacement of conventional electronic signal in data processor is mainly because of the inherent parallelism in optics, which can lead to a super fast up-gradation of computing technology. The major developments conducted in this area are also discussed in the 1st and 2nd International Conferences on Photonic Computing. ${ }^{12,13}$

In connection to the new developments of several all-optical data processing techniques, the role of optical tree architecture can be mentioned specially. This tree has already been used to convert a position-wise encoded optical decimal data to its binary counterpart and from binary data to its decimal counterpart. ${ }^{14}$ Not only these conversions but there are also several other conversions which are possibly conducted by the use of this tree architecture for 
the purpose of all-optical data processing and computing. Here in this communication, the authors propose a modification of optical tree architecture by which a binary data having a fractional part can be converted to its respective equivalent decimal value and from decimal number having a fractional part to its equivalent binary form. Already optical tree has been used for conversion of different forms of data, but the optical conversion of binary number having fractional part to its decimal counterpart and its vice-versa is a completely new concept.

\section{Optical Tree-Architecture}

The schematic diagram of an optical tree-architecture is shown in Fig. 1. Here a light beam (preferably a laser) is emitted from a point source (A). Then the light beam passes through some optical channels. Here each channel is divided into two different light paths e.g. AB into $\mathrm{BC}$ and $\mathrm{BD}$ by the use of some beam splitters. Each of $\mathrm{BC}$ and $\mathrm{BD}$ is divided into two more parts individually as $\mathrm{BC}$ to $\mathrm{CE}$ and $\mathrm{CF}$ and $\mathrm{BD}$ to $\mathrm{DG}$ and $\mathrm{DH}$. Proceeding in this way ultimately eight spots (from I to P) are obtained from a single beam AB. By arranging another set of splitting arrangement in the output plane one can get 16 spots. To use this circuit as a data processor, one can use some optical channels $\left(\mathrm{A}_{0}, \mathrm{~A}_{1}, \mathrm{~A}_{2}\right)$ which can control the way of passing of the light beam from one main channel to a sub channel. These control channels also carries light beam. $\mathrm{A}_{2}$ control channel is connected to the switching point $\mathrm{B}$ and $\mathrm{A}_{1}$ channel is connected to $\mathrm{C}$ and $\mathrm{D}$ switching points. $\mathrm{A}_{0}$ channel is connected to E, F, G, H switches. The function of the switches can be illustrated by an example. If light in the control channel $\mathrm{A}_{2}$ is present then it activates the switch $\mathrm{B}$ to pass the light from $\mathrm{AB}$ to $\mathrm{BC}$ (upper) sub channel otherwise the light of $\mathrm{AB}$ channel will go through the (lower) sub channel BD. The other switches operate in the same way. One can use this optical system for the conversion of a binary number having a fractional part

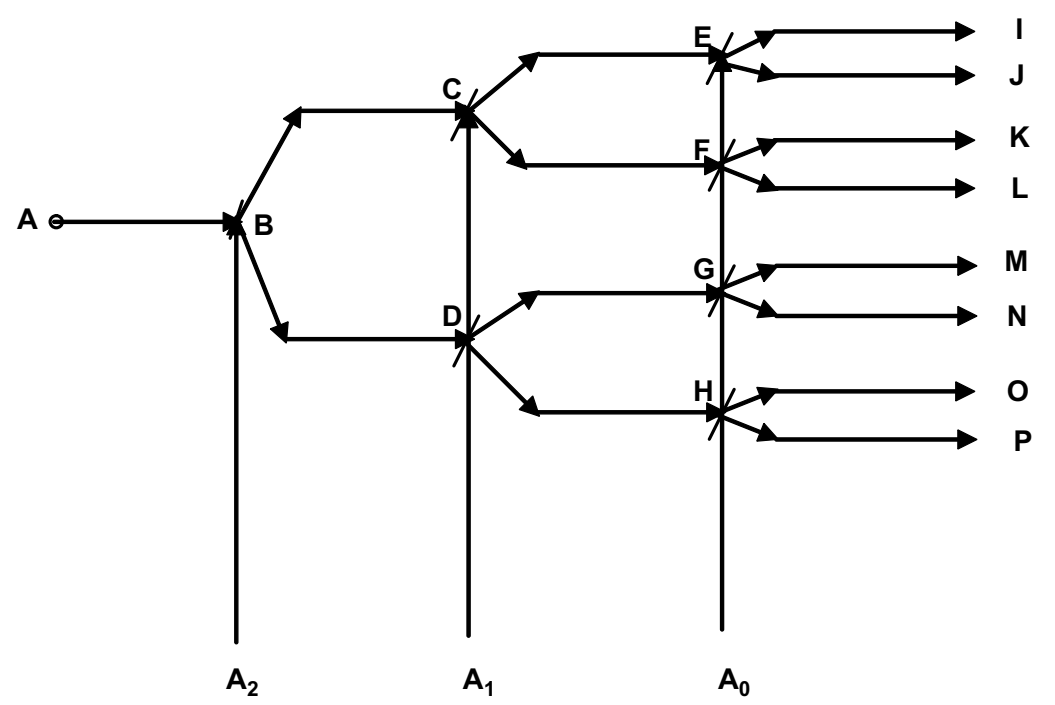

Fig. 1. Optical tree architecture for binary to decimal conversion. 
to its equivalent decimal number. In the present analysis, all the switches are all-optical in nature. Now for the conversion of a fractional decimal number to its binary equivalent system, a different arrangement of mirrors, beam splitters are needed.

\section{Non-Linear Material as an Optical Switch}

The Kerr type of non-linear materials like $\mathrm{CS}_{2}$, pure silica glass etc can show its isotropic non-linear behavior of the refractive index of the material for passing the light through it. Due to this behavior, the refractive index of the material $(n)$ maintains the equation as $n=n_{0}+n_{2} \mathrm{I}$, where $n_{0}$ is a constant refractive index term, $n_{2}$ is a non-linear correction term and $\mathrm{I}$ is the intensity of the light passing through it. According to this equation, when a beam $\mathrm{AB}$ of some fixed intensity $\mathrm{I}$ falls in the point $\mathrm{O}(\mathrm{O}$ is a point in the interface of a linear material (LM) and non-linear material (NLM)), it then passes through the DE channel as shown in Fig. 2. Now when one beam of intensity I in AB channel and other one beam of intensity I in CMB channel fall jointly on "O" , then according to the above equation, the non-linear refractive index of the material becomes $n=n_{0}+2 n_{2} \mathrm{I}$, and for this reason light beam ultimately will pass through the FG channel of Fig. 2. Thus the behavior of the NLM can be compared as an optical switch. Already many all-optical logic devices have been proposed based on this principle. ${ }^{15-18}$

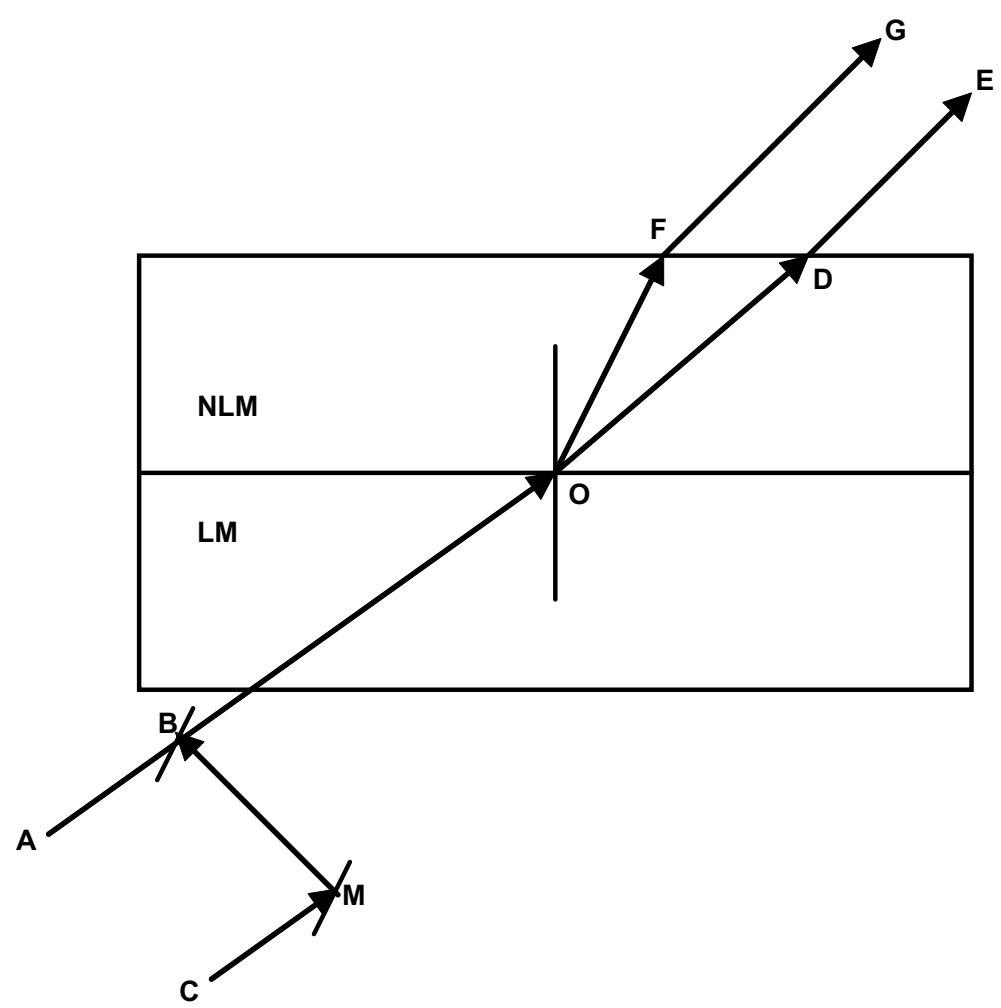

Fig. 2. Non-linear material as an optical switch. 


\section{Optical Conversion Method of a Binary Number Having a Fractional Part to Its Equivalent Decimal Number}

The optical method of conversion of binary to decimal number is an established one. ${ }^{14}$ The next important function is the implementation of a system for transformation of a fractional binary number to its decimal counterpart. Here modified tree architecture can be used for such conversion. The significant difference of this scheme from that described earlier is the method of placement and the position of NLM based switches, beam splitters (BS) and mirrors $(\mathrm{M})$. Placing these components in a properly different way, one can develop the modified system. The whole scheme is shown in Fig. 3 . Here the control channels $\left(\mathrm{B}_{4} \mathrm{~B}_{3} \mathrm{~B}_{2} \mathrm{~B}_{1} \mathrm{~B}_{0}\right)$ carry the bits of fractional binary number. The whole things of the system can be made clear by an example. Let the fractional binary be 0.10110. So $\mathrm{B}_{4}=\mathrm{B}_{2}=\mathrm{B}_{1}=1$ and $\mathrm{B}_{3}=\mathrm{B}_{0}=0$ and for these type of control/input signals, the beam originally generated from a constant light source (CLS) first passes through the upper channel from $\mathrm{NLM}_{1}$ then it comes to the lower channel from $\mathrm{NLM}_{2}$ and again passing through the upper channel from $\mathrm{NLM}_{4}$ it ultimately exits through the channel number 7 . This indicates that the conversion of 0.10110 gives the position-wise encoded decimal number 0.7. In this way, any binary number having a fractional part can be converted to the respective decimal number by the use of the system described in a Fig. 3. Now the whole scheme can be integrated together to convert a binary number having both fractional and non-fractional parts to its equivalent decimal value. This integrated scheme is shown in Fig. 4. Here the block-A consists of the

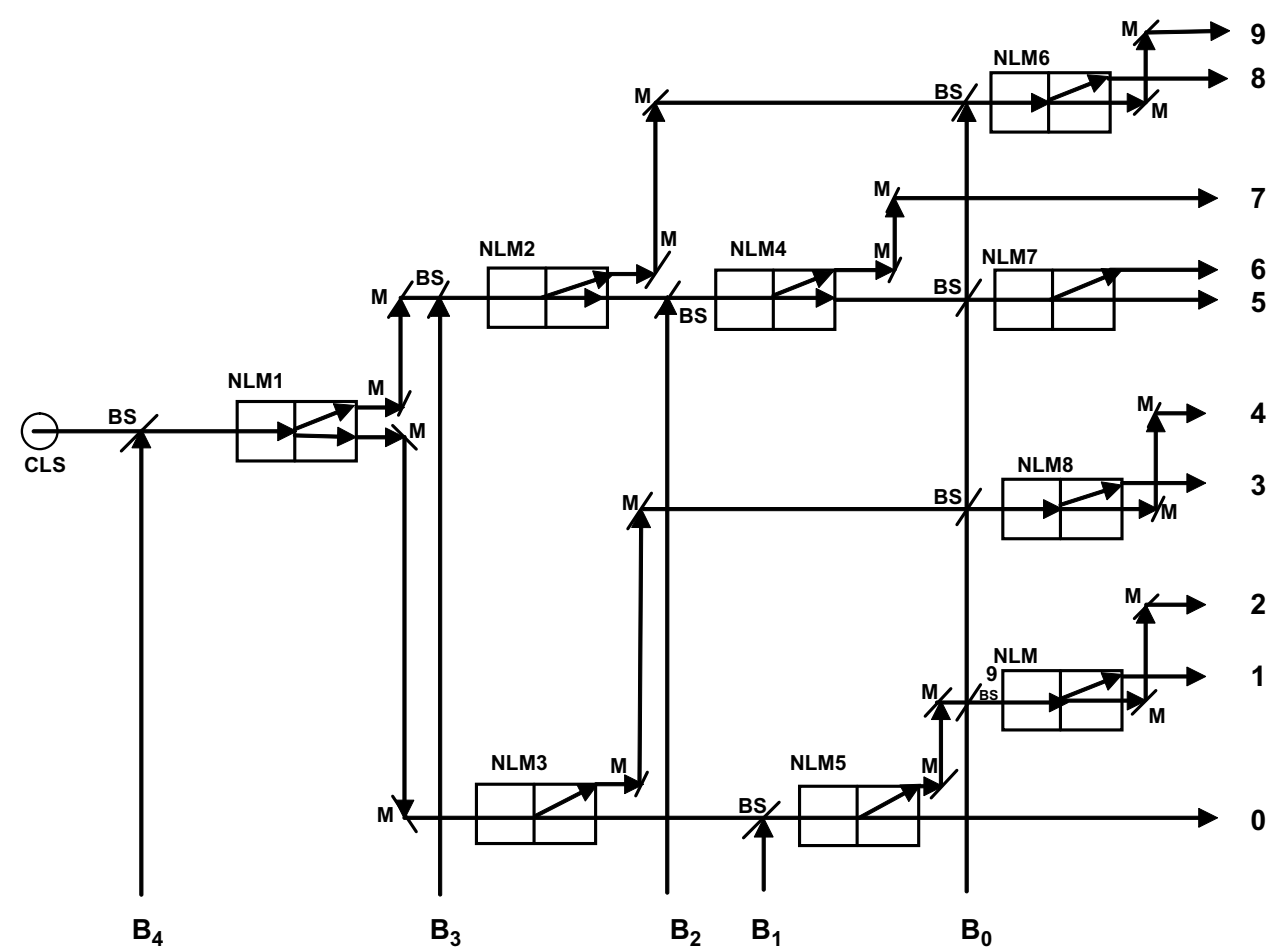

Fig. 3. All optical system for converting fractional binary number to its decimal counterpart. 


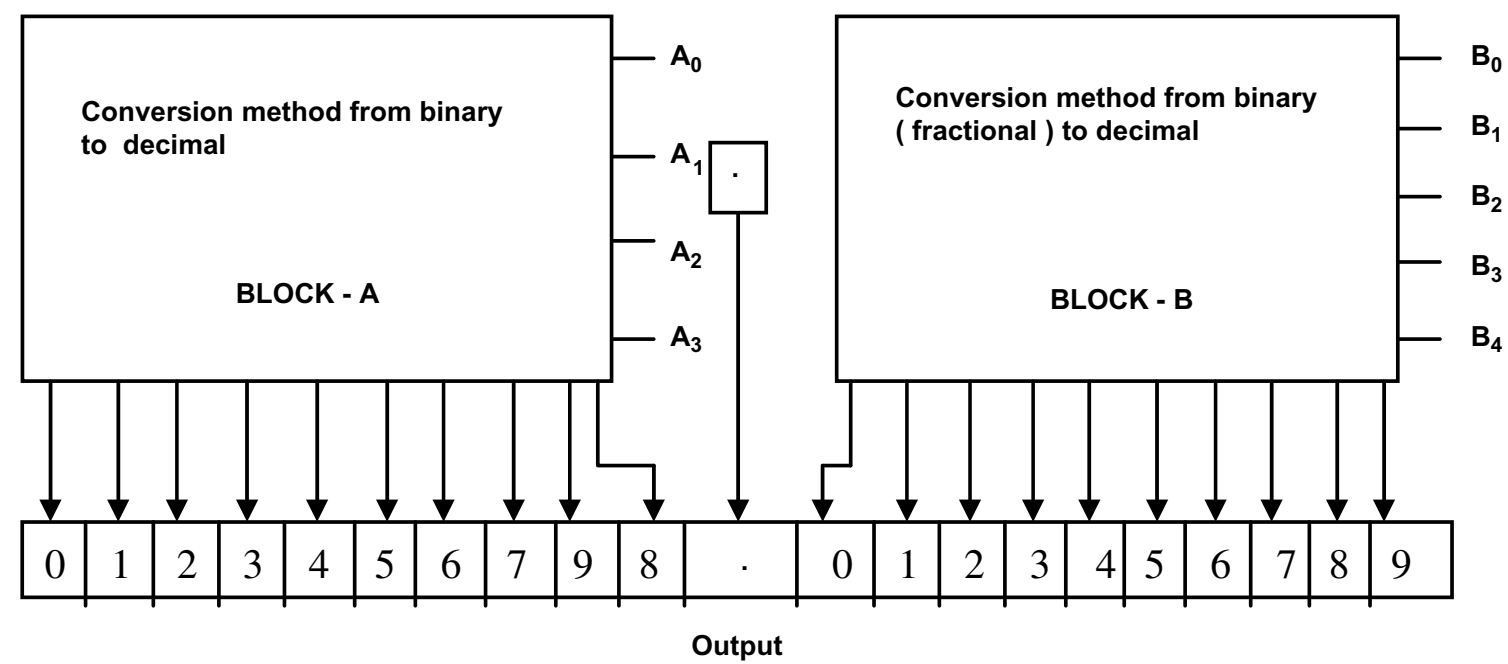

Fig. 4. All optical integrated system for converting a binary number having both fractional part and nonfractional part to its equivalent decimal number.

system of non-fractional part. ${ }^{14} \mathrm{~A}_{3} \mathrm{~A}_{2} \mathrm{~A}_{1} \mathrm{~A}_{0}$ are the input channels i.e. the channels for placing the binary non-fractional inputs. On the other hand block-B carries the same system as described in Fig. 3 i.e. fractional part, where $\mathrm{B}_{4} \mathrm{~B}_{3} \mathrm{~B}_{2} \mathrm{~B}_{1} \mathrm{~B}_{0}$ are the input channels for placing the binary fractional inputs. By the joint action of the two blocks (A and $\mathrm{B}$ ) one can convert any binary number (having both fractional and non-fractional parts) to its equivalent decimal value which is encoded position-wise. For example if 011.10011 are applied to this integrated system, one can obtain 3.6 at the output. All the converted fractional decimal numbers from its equivalent binary numbers are shown in Table 1.

\section{Optical Conversion Method of a Decimal Number Having a Fractional Part to Its Binary Equivalent}

Now we also propose a concept of converting a decimal number having a fractional part to its binary equivalent in an all optical process. Here the decimal number has two parts as a whole. First part is non fractional ${ }^{14}$ whereas second part is the fractional one. The second part extends the conversion of fractional part. The proposed system for conversion of the fractional digit to its binary equivalent is shown in Fig. 5. Here we use beam combiners for coupling two light beams into a single one and also for breaking a single beam into two parts. These are represented by BS in the figure. The mirrors (M) are used for the reflection of light beam. Using these $\mathrm{BSs}$, one can get the converted result at the output channel $\mathrm{B}_{4} \mathrm{~B}_{3} \mathrm{~B}_{2} \mathrm{~B}_{1} \mathrm{~B}_{0}$ of the system shown in Fig. 5. By the use of the modified tree structure, ultimately one can show the converted equivalent binary number of the fractional decimal contribution, which lies from 0 to 0.9 in the channels $\mathrm{B}_{4} \mathrm{~B}_{3} \mathrm{~B}_{2} \mathrm{~B}_{1} \mathrm{~B}_{0}$. To develop this system, the channels marked by $0,1,2,3, \ldots, 9$ are used for applying the fractional part of the decimal number. If 0.8 is to be converted, then the light should be applied at the channel marked by 8 , 


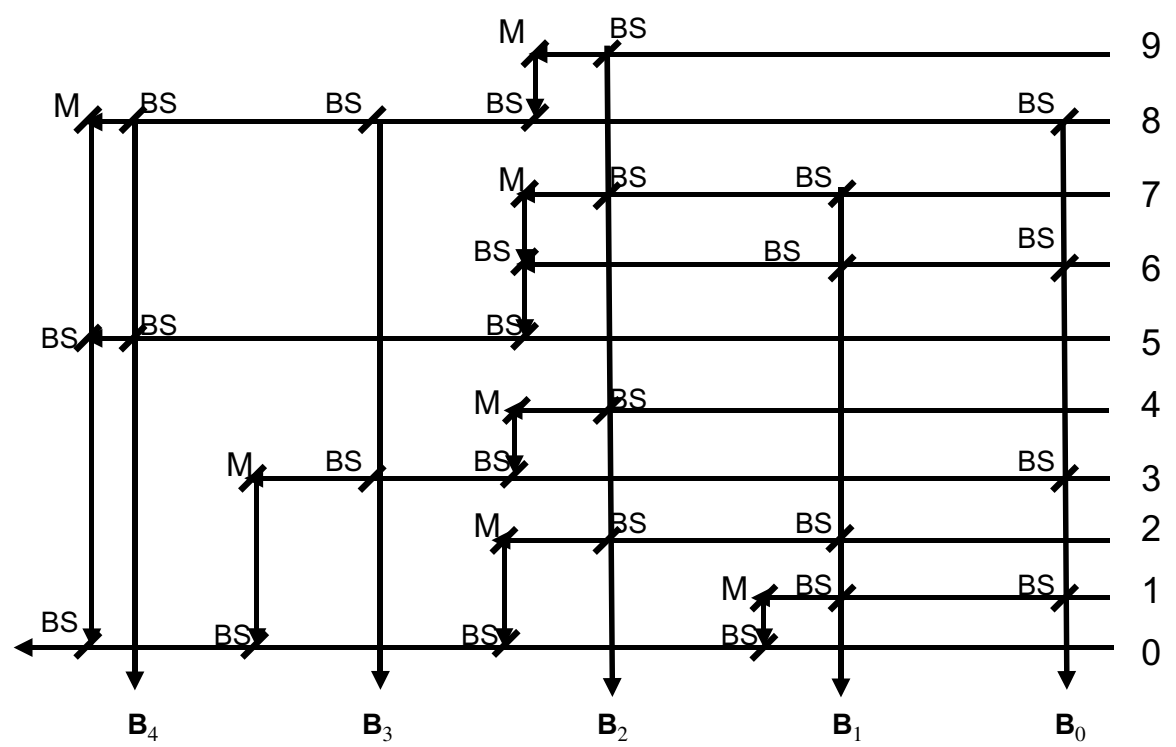

Fig. 5. All-optical conversion system from fractional decimal number to its binary counter part.

and the converted binary output is obtained in the channels marked by $\mathrm{B}_{4} \mathrm{~B}_{3} \mathrm{~B}_{2} \mathrm{~B}_{1} \mathrm{~B}_{0}$. As an example, when the light goes through the channel 7, no light comes into the channels $\mathrm{B}_{0}$ and $\mathrm{B}_{3}$ because of the absence of $\mathrm{BS}$ in the respective channel. So $\mathrm{B}_{0}=\mathrm{B}_{3}=0$, but $\mathrm{B}_{4}, \mathrm{~B}_{2}, \mathrm{~B}_{1}$ channels get the necessary light reflected from the respective BS. Hence $\mathrm{B}_{4}=\mathrm{B}_{2}=\mathrm{B}_{1}=1$. Ultimately one can obtain $\mathrm{B}_{4} \mathrm{~B}_{3} \mathrm{~B}_{2} \mathrm{~B}_{1} \mathrm{~B}_{0}=10110$ for the application of light in the input channel marked by 7 . So it is the equivalent binary number of decimal value 0.7 . Similarly, any decimal number from 0 to 0.9 can be converted to its binary value. The non-fractional part and fractional part of a system can be combined together to develop a single integrated system from which one can convert a decimal number having both the non fractional part and the fractional part to its binary equivalent value. The scheme is shown in Fig. 6. As an example, if it is required to convert the decimal number a.b to its equivalent binary number (where ' $a$ ' is the non fractional decimal digit and ' $b$ ' is the fractional decimal digit), the light beams are to be placed in the 'a'th channel of the block A and to ' $\mathrm{b}$ 'th channel of the block B in the system described in Fig. 6. Here block A and block $\mathrm{B}$ comprise the system of non-fractional part and the system of fractional part respectively. The $\mathrm{A}_{3} \mathrm{~A}_{2} \mathrm{~A}_{1} \mathrm{~A}_{0} \cdot \mathrm{B}_{4} \mathrm{~B}_{3} \mathrm{~B}_{2} \mathrm{~B}_{1} \mathrm{~B}_{0}$ comes as the result of the converted binary number from its decimal counterpart. If the decimal number is 3.7, one can surely receive 0011.10110 at the output stage i.e. $A_{1}=A_{0}=B_{4}=B_{2}=B_{1}=1$ and $A_{3}=A_{2}=B_{3}=B_{0}=0$.

\section{Alternating Approach of Non-Linear Switch}

In this communication, we have used Kerr type of non-linear material as an optical switch for conducting the intensity encoded data based optical conversion. In addition to this method, we can propose the use of an alternative optical switch, which can be realized by 


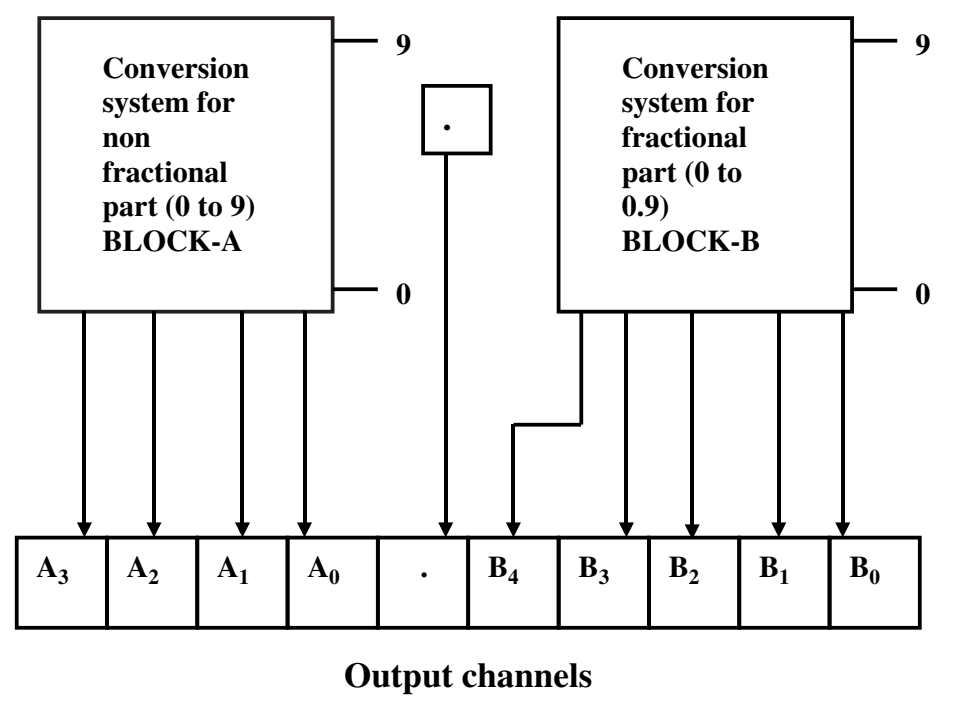

Fig. 6. The block diagram of complete system for conversion of decimal number having both fractional part and non-fractional part to its binary equivalent.

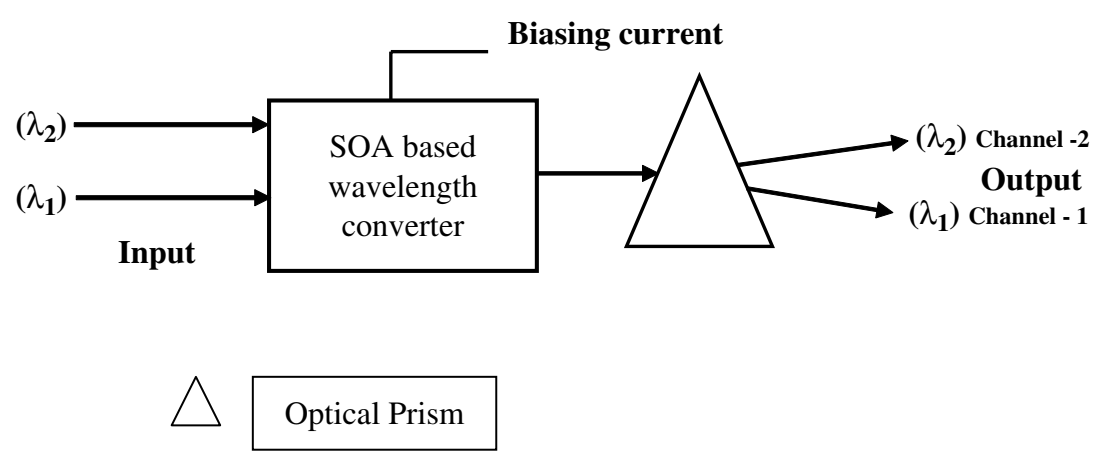

Fig. 7. SOA based intensity encoded switch.

the use of non-linear character of semiconductor optical amplifier (SOA). Here the channel shifting operation in the tree architecture is conducted by SOA. In this process, one strong pump beam of $\lambda_{1}$ wavelength is applied into the input terminal of a properly biased SOA based wavelength converter (Fig. 7). One prism is placed in front of it. So $\lambda_{1}$ wavelengths of light first passing through the SOA switch goes through the prism and exits from a specific (channel 1). Now if a weak probe beam ( $\lambda_{2}$ wavelength) is applied to the input side of the SOA switch in addition to the existing pump beam, then one can get a converted light beam of wavelength $\lambda_{2}$ from the output of the SOA switch and after passing through the prism, it comes out through another channel (channel 2). Thus two light beams come in two different channels of the output in the system described in Fig. 7. When a light of wavelength $\lambda_{1}$ is applied at the input side of the SOA, it ultimately exits from channel-1 and when light of $\lambda_{1}$ and $\lambda_{2}$ wavelengths both falls in the input side of SOA, one gets light from another 
channel marked as channel-2. This type of switch can be used as replacement of the switch shown in Fig. 2. It is already seen that for the conversion of a decimal number (having some fractional part) to the equivalent binary number the system proposed in Fig. 3 and Fig. 4 will be useful with this alternative switch. The conventional non-linear switches used in these two systems may be replaced by the SOA based switches as described above to get a faithful operation. The output power of the digits of the converted data becomes stronger here sufficiently.

In the case of decimal (with fractional number) to binary conversion, no optical switches are required.

\section{Conclusion}

To conclude, the method of conversion of a binary number having a fractional part to its equivalent decimal number and its vice-versa with all optical switching system have been discussed here. The speed of operation is real time. The scheme may be extended both vertically as well as horizontally for the conversion of a higher valued binary number to its equivalent decimal number and also from decimal to binary. The inherent parallelism of optics is exploited in the scheme as far as practicable to obtain superfast operation speed. To get more accuracy in the conversion of the fractional binary number, one may use a greater number of control channels in an extended system. As a whole, the total system is an all optical one and hence the advantages of using optics are achieved. We should use a suitable non-linear material like SOA and a suitable diode laser, for getting low optical power consumption, superfast speed of operation and for reliable operational result.

\section{References}

1. M. A. Karim and A. S. Awwal, Optical Computing An Introduction (John Wiley and Sons, Canada, 1992).

2. M. A. Karim, A. A. S. Awwal and A. K. Cherri, Polarisation-Encoded Optical Shadow-Casting Logic Unit:Design, Appl. Opt. 26 (1987) 2720-2725.

3. T. Houbavlis and K. Zoiros, 10-GHZ all-optical recirculating shift register with semiconductor optical amplifier (SOA)-assisted sagnac switch and SOA feedback, Opt. Engg. 42(9) (2003) $2483-2484$.

4. K. R. Chowdhury, A. Sinha and S. Mukhopadhyay, An all optical comparison scheme between two multi-bit data with optical nonlinear material, Chinese Optics Letters 6(9) (2008) 693.

5. K. R. Chowdhury, P. P. Das and S. Mukhopadhyay, All-optical time-domain multiplexingdemultiplexing scheme with non-linear material, Optical Engineering 44(3) (2005) 035201.

6. M. T. Hill, H. de Waardt, G. D. Khoe and H. J. S. Dorren, Fast optical flip-flop by use of Mach-Zehnder interferometers, Microwave Optical Technology Letters 31 (2001) 411-415.

7. M. T. Hill, H. de Waardt, G. D. Khoe and H. J. S. Dorren, All-optical flip-flop based on coupled laser diodes, Journal of Quantum Electronics 37 (2001) 405-413.

8. T. Houbavlis and K. Zoiros, 10-GHZ all-optical recirculating shift register with semiconductor optical amplifier (SOA)-assisted sagnac switch and SOA feedback, Opt. Engg. 42(9) (2003) $2483-2484$.

9. S. Dutta and S. Mukhopadhyay, An all optical approach of frequency encoded NOT based Latch using semiconductor optical amplifier, Journal of optics 39(1) (2010) 35-41. 
10. S. Dutta and S. Mukhopadhyay, All optical frequency encoding method for converting a decimal number to its equivalent binary number using tree architecture, Optik-Int. J. Light Electron Opt. (2010), 10.1016/j.ijleo.2009.11.018.

11. S. Dutta and S. Mukhopadhyay, Alternating approach of implementing frequency encoded alloptical logic gates and flip-flop using semiconductor optical amplifier, Optik-Int. J. Light Electron Opt. (2010), doi:10.1016/j.ijleo.2010.06.046. Article in press.

12. "Optical super computing", First International Workshop, OSC 2008 Vienna Austria, August 26, 2008 proceedings, Published by Springer Berlin/Heidelberg, Volume 5172/2008.

13. "Optical super computing", Second International Workshop, OSC 2009 Bertinoro, Italy, November 18-20, 2009 Proceedings.

14. S. Mukhopadhyay, An optical conversion system: From binary to decimal and decimal to binary, Optics Communications (The Netherlands) 76(5-6) (1990) 309-312.

15. F. T. S. Yu, Q. W. Song and X. J. Lu, Implementation of Boolean logic gates using a macrochannel spatial light modulator with liquid-crystal television, Optics Lett. 12 (1987) 962.

16. F. T. S. Yu, S. Jutamulia and D. A. Gregory, Optical parallel logic gates using inexpensive liquid crystal television, Optics Lett. 12 (1987) 1050.

17. K. M. Jhonson, M. R. Surette and J. Samir, Optical interconnection network using polarizationbased ferroelectric liquid crystal gates, Appl. Optics 27 (1988) 1727.

18. T. Sato, K. Shiraishi, K. Tsuchida and S. Kawakami, Laminated polarization splitter with a large split angle, ApPhL. 61 (1992) 2633S. 